\title{
Phytochemical Composition and Chronic Hypoglycemic Effect of Bromelia karatas on STZ-NA-Induced Diabetic Rats
}

\author{
Sonia M. Escandón-Rivera, Adolfo Andrade-Cetto (D), and Gabriela Sánchez-Villaseñor \\ Laboratorio de Etnofarmacología, Facultad de Ciencias, Universidad Nacional Autónoma de México, 4510 Ciudad de México, Mexico \\ Correspondence should be addressed to Adolfo Andrade-Cetto; aac@ciencias.unam.mx
}

Received 25 October 2018; Revised 17 December 2018; Accepted 11 February 2019; Published 25 February 2019

Academic Editor: Daniela Beghelli

Copyright (C) 2019 Sonia M. Escandón-Rivera et al. This is an open access article distributed under the Creative Commons Attribution License, which permits unrestricted use, distribution, and reproduction in any medium, provided the original work is properly cited.

\begin{abstract}
Oral administration of an aqueous extract of the aerial parts of Bromelia karatas to STZ-NA rats showed a significant hypoglycemic effect in a chronic trial lasting 42 days. Chromatographic profiles of the active extract (WE) and an organic extract (OE) of $B$. karatas were obtained by high-performance liquid chromatography (HPLC) and used to identify their major components. Isolation and identification of the compounds present in the extracts were accomplished by means of various conventional chromatographic and spectroscopic techniques. This process led to the identification of $\beta$-sitosterol-3-O- $\beta$-D-glucopyranoside $(\mathbf{1})$ and $\rho$-coumaric acid (3) as the major compounds present in the extracts. During the isolation of $\mathbf{1}$ and $\mathbf{3}$, seven additional metabolites not previously reported for the plant were obtained, namely, cirsiliol $4^{\prime}-\mathrm{O}-\beta$-D-glucopyranoside (2), stigmasterol (4), $\beta$-sitosterol (5), 1-O-feruloyl3-O- $\rho$-coumaroylglycerol (6), $\beta$-D-(1-O-acetyl-3,6-O-trans-diferuloyl) fructofuranosyl- $\alpha$-D-2' $4^{\prime}, 6^{\prime}$-O-triacetylglucopyranoside (7), 1-O-p-coumaroyl-3-O-caffeoylglycerol (8), and 2-propyl- $\beta$-glucopyranoside (9).
\end{abstract}

\section{Introduction}

Diabetes mellitus is a metabolic disease that is characterized by elevated levels of blood glucose that over time cause serious damage to the heart, blood vessels, eyes, kidneys, and nerves $[1,2]$. Individuals with Type 2 diabetes typically suffer from insulin resistance and relative rather than absolute insulin deficiency. At least initially, and often throughout their lifetimes, these individuals may not require insulin treatment to survive. According to the IDF 2017, 425 million adults have diabetes worldwide, and Mexico ranks 6th among the top ten countries in the number of adults with diabetes.

In Mexico, the use of plants for medicinal purposes has been a common practice since pre-Hispanic times. Among the plants used in the treatment of diabetes, Bromelia karatas (L) traditionally known as "piñuela" "chiyol", "chicipo", "aguama", and "cazuela" is a tropical herb that is distributed between 400 and $1500 \mathrm{~m}$ elevation. The leaves are elongated and thick and contain sharp teeth along the margins. Inflorescence is sessile at the ground level. Flowering of the plant occurs between May and October. Its distribution in America ranges from Mexico to Ecuador and Brazil; in
Mexico, it is mainly found in the tropical zone [3]. B. karatas is a monocotyledon that produces a variety and diversity of antioxidant metabolites and glucoconjugates such as $(6 \mathrm{~S}, 9 \mathrm{R})$ vomifolyl- $\beta$-D-glucopyranoside, 3,4,5-trimethoxyphenyl- $\beta$ D-glucopyranoside, 1-O- $\beta$-D-glucopyranosyl anthranilate, and 3,4-dimethoxyphenyl $\beta$-D-glucopyranoside [4]. In a previous work, we reported the traditional use of the plant in the treatment of diabetes, and its acute hypoglycemic effect was demonstrated in STZ-NA hyperglycemic rats [5]. That investigation showed that diabetic patients and healers in the municipality of Tlanchinol, Hidalgo, use a decoction of the leaves to control blood glucose levels and confirmed the hypoglycemic effects of aqueous and hydroalcoholic extracts of the plant; these extracts produced the best effects at oral doses of 350 and $300 \mathrm{mg} / \mathrm{kg}$, respectively. The study also reported preliminary experiments to determine the presence of alkaloids, terpenes, and phenolic compounds in the extracts and found that phenolic compounds are the main components. However, to date, the metabolites present in the active extract of $B$. karatas have not been identified.

The aim of the current study was to continue trials of the hypoglycemic effect of administration of a water extract of 
leaves of B. karatas in STZ-NA rats and to evaluate the lipid profiles and glycated hemoglobin levels of the animals after chronic administration of the extract. An additional aim of the study was to characterize the main components of the extract using conventional phytochemical techniques for the separation, isolation, and identification of metabolites.

\section{Materials and Methods}

2.1. General Experimental Procedures. NMR spectra including ${ }^{1} \mathrm{H},{ }^{13} \mathrm{C}$, HSQC, HMBC, COSY, NOESY, and TOCSY were recorded in a Varian Inova spectrometer at $400\left({ }^{1} \mathrm{H}\right)$ and $95 \mathrm{MHz}\left({ }^{13} \mathrm{C}\right)$ or a Bruker DMX500 spectrometer operated at $500 \mathrm{MHz}\left({ }^{1} \mathrm{H}\right)$ or $125 \mathrm{MHz}\left({ }^{13} \mathrm{C}\right)$; chemical shifts were recorded as $\delta$ values. ESI-MS were recorded on a Thermo Scientific LTQ Orbitrap XL hybrid FTMS (Fourier transform mass spectrometer). Data were collected in both positive and negative ionization modes via a liquid chromatographic/autosampler system that consisted of an Agilent HPLC system. Analytical and preparative HPLC analyses were performed in an Agilent 1260 Infinity system equipped with a G1311B Quaternary Pump, a G1367E Autosampler, and a G1315C DAD VL+ and controlled by Agilent ChemStation software. For analytical and semipreparative HPLC, Phenomenex (Luna Omega Polar $\mathrm{C}_{18}, 50 \times 2.1 \mathrm{~mm}$ id., $1.6 \mu \mathrm{m}$ ) and Macherey-Nagel (Nucleosyl $\mathrm{C}_{18}, 250 \times 4.6 \mathrm{~mm}$ id., $5 \mu \mathrm{m}$ and Nucleosyl $\mathrm{C}_{18}, 250 \times 10 \mathrm{~mm}$ id., $\left.5 \mu \mathrm{m}\right)$ columns, respectively, were used. Column chromatography (CC) was conducted on silica gel (70-230 mesh, Merck) or Sephadex LH-20 (Sigma-Aldrich Chemical). Thin-layer chromatography analyses were carried out on silica gel $60 \mathrm{~F}_{254}$ plates (Macherey \& Nagel) using ceric sulfate (10\%) solution in $\mathrm{H}_{2} \mathrm{SO}_{4}$ as the color reagent.

2.2. Plant Extracts. The original plant material was collected with the help of the healer "Isabel Escalante" near the town of Tamala, state of Hidalgo, Mexico. The plant species was verified by MC Ramiro Cruz Durán, and a voucher exemplar was deposited at the Herbarium of the "Instituto Mexicano del Seguro Social" (IMSSM 15814). Fresh plant material was collected as needed.

The water extract (WE) was prepared by boiling $20 \mathrm{~g}$ of the dry plant material with $500 \mathrm{ml}$ water, followed by filtration and lyophilization to yield $2.119 \mathrm{~g}$ of WE. The extract was stored at $4^{\circ} \mathrm{C}$ for further analysis. The WE and the organic extract $(\mathrm{OE})$ were used for phytochemical identification of the main compounds of $B$. karatas. OE was prepared from $365 \mathrm{~g}$ of plant material by consecutive extraction with a mixture of dichloromethane:methanol (1:1). The combined extract was concentrated in a rotary vacuum evaporator (Buchi, Flawil, Switzerland) at $40^{\circ} \mathrm{C}$; the resulting concentrate was evaporated to dryness under reduced pressure, yielding $8.42 \mathrm{~g}$ of $\mathrm{OE}$.

2.3. Experimental Animals. Wistar rats weighing 200-250 g were provided by the Bioterium of the Science School, UNAM; they were acclimated with free access to food and water for at least one week in an air-conditioned room $\left(25^{\circ} \mathrm{C}\right.$,
$50 \%$ humidity) on a 12 -h light-dark cycle prior to use in the experiments. All the experiments were conducted in accordance with the principles set forth in the National Institute of Health (NIH) Guidelines for the Care and Use of Laboratory Animals [6]. Hyperglycemia was induced as described by Masiello [7]. In brief, rats that had been fasted overnight were injected intraperitoneally with $150 \mathrm{mg} / \mathrm{kg}$ nicotinamide (NA) (Sigma, N3376) 15 min prior to intravenous injection of $65 \mathrm{mg} / \mathrm{kg}$ streptozotocin (STZ, Sigma S0130) in citrate buffer. Hyperglycemia was identified by polyuria and polydipsia and by measuring nonfasting plasma glucose levels $48 \mathrm{~h}$ after the injection; animals that did not reach glucose levels of $250 \mathrm{mg} / \mathrm{dl}$ were rejected.

Experiment 1. Chronic Hypoglycemic Effect of the Water Extract. The previously described experimental design [8] was used; in brief, four groups of six rats were used as follows: group I, the normal control (NC), received $1.5 \mathrm{ml}$ of physiological $\mathrm{NaCl}$ solution (vehicle); group II, the hyperglycemic control (HC), also received $1.5 \mathrm{ml}$ of physiological $\mathrm{NaCl}$ solution; group III was given a standard oral hypoglycemic agent, glibenclamide [ $5 \mathrm{mg} / \mathrm{kg}$ body weight (bw)] in the same vehicle (CG); and group 4 received Bk-WE $(218 \mathrm{mg} / \mathrm{kg}$ bw) dissolved in $1.5 \mathrm{ml}$ of physiological $\mathrm{NaCl}$ solution. The extract or the hypoglycemic agent was orally administered twice daily (in the morning and in the evening) over a period of 42 days. All groups were fed Purina Rodent Laboratory Chow 5001 .

Blood was obtained from the tail veins of the animals; the animals were handled according to the procedures described in [6]. Glucose monitoring was performed weekly using glucose test strips and an Accutrend Plus ${ }^{\circledR}$ glucometer. Glycated hemoglobin (HbAlc) was analyzed in a DCA Vantage ${ }^{\circledR}$ Siemens. The lipid profile (HDL, TG and cholesterol) was measured using Cardio Check $^{\circledR}$ and Panels ${ }^{\circledR}$ PTS strips. VLDL was calculated using the following equation: VLDL = $0.2 \mathrm{x}$ TG. HbAlc and lipid profiles were measured on days 0 , 14,28 , and 42 after the initiation of treatment. The data were statistically analyzed using the unpaired t-test. The plasma glucose levels are expressed as the mean (S.E.M.).

Experiment 2. Acute Hypoglycemic Effect of the Isolated Compounds. To know if the activity is based on the presence of compounds $1-3$, or on the presence of any of them in the WE, an acute experiment was performed as previously described [8]. Wistar rats were divided into six groups of four rats each. Group 1, normal control, orally received $1.5 \mathrm{ml}$ of physiological $\mathrm{NaCl}$ solution (vehicle), group 2, hyperglycemic control, also received $1.5 \mathrm{ml}$ of physiological $\mathrm{NaCl}$ solution, group 3, positive control, received a standard oral hypoglycemic agent [glibenclamide, $5 \mathrm{mg} / \mathrm{kg}$ body weight (bw)] in the same vehicle. Groups 4, 5, and 6 were given compounds $1(72 \mathrm{mg} / \mathrm{kg}), 2(1.8 \mathrm{mg} / \mathrm{kg})$, and $3(3.63 \mathrm{mg} / \mathrm{kg})$ all dissolved in $1.5 \mathrm{ml}$ of physiological $\mathrm{NaCl}$ solution. The doses of each compound were calculated according to the radio; plant: yield of the compound.

2.4. HPLC Analysis. Elution was conducted at a flow rate of $0.25 \mathrm{~mL} / \mathrm{min}$ with water containing $0.1 \%$ formic acid as 
solvent $\mathrm{A}$ and acetonitrile $(\mathrm{MeCN})$ as solvent $\mathrm{B}$, using a gradient elution of 100:0 (A:B) for $1 \mathrm{~min}, 95: 5(\mathrm{~A}: \mathrm{B})$ at $1.5 \mathrm{~min}$, 85:15 (A:B) at 1.5-12 $\mathrm{min}, 70: 30(\mathrm{~A}: \mathrm{B})$ at 12-20 $\mathrm{min}, 70: 30(\mathrm{~A}: \mathrm{B})$ maintained during $1 \mathrm{~min}, 60: 40(\mathrm{~A}: \mathrm{B})$ at $21-23 \mathrm{~min}, 20: 80$ $(\mathrm{A}: \mathrm{B})$ at $23-27 \mathrm{~min}, 10: 90(\mathrm{~A}: \mathrm{B})$ at $27-28 \mathrm{~min}, 10: 90(\mathrm{~A}: \mathrm{B})$ maintained during $1 \mathrm{~min}$, and 100:0 (A:B) at 29-31 min. The flow rate was set to $0.25 \mathrm{~mL} / \mathrm{min}$, and the temperature of the column was maintained at $35^{\circ} \mathrm{C}$. System control, data collection, and data processing were accomplished using OpenLAB LC 1260 chromatography software. Working solutions of samples (WE, OE, fractions and isolated compounds) of $B$. karatas were prepared by dissolving $5.0 \mathrm{mg}$ of the sample in $1 \mathrm{~mL}$ of the appropriate solvent $\left(\mathrm{MeOH}, \mathrm{MeCN}\right.$ or $\left.\mathrm{H}_{2} \mathrm{O}\right)$ and injected $(2 \mu \mathrm{L})$ using an autosampler. For UV detection, the wavelength program was set at an acquisition of $\lambda 230,254$, 280,320 , and $365 \mathrm{~nm} ; 320 \mathrm{~nm}$ was selected as the optimum wavelength. The equipment, data acquisition, processing, and management of the chromatographic information were controlled with the OpenLab CDS ChemStatiob Edition (2001-2013) software. The separation was carried out using a Phenomenex ${ }^{\circledR}$ Luna Polar C18 $(50 \times 2.1 \mathrm{~mm}$ id., $1.6 \mu \mathrm{m})$ reverse phase column; all solvents were purchased from JT Baker as HPLC grade.

2.5. Isolation Compounds. WE $(1 \mathrm{~g})$ was dissolved in a mixture of $\mathrm{MeOH}: \mathrm{H}_{2} \mathrm{O}$ (1:1); from this solution, $241 \mathrm{mg}$ of 1 spontaneously precipitated. The remainder of the WE was further separated on Sephadex (500 mL MeOH: $\mathrm{H}_{2} \mathrm{O}$ 1:1), yielding 14 primary fractions (WE1-WE14). WE9 (43 mg) was resolved by HPLC (Nucleosil $250 \times 10 \mathrm{~mm}$ i.d., $5 \mu \mathrm{m}$, C18, MachereyNagel) using a 15-min gradient of MeCN:MeOH: $\mathrm{H}_{2} \mathrm{O}$ $(60: 5: 35)(2.3 \mathrm{ml} / \mathrm{min} ; 254$ and $320 \mathrm{~nm})$, yielding $6 \mathrm{mg}$ of a solid yellow amorphous powder (2) with $\mathrm{R}_{\mathrm{T}}: 9.53 \mathrm{~min}$. WE13 (46.5 mg) was subjected to preparative TLC (EtOAc:nhexane:acetone 7:2:1) to yield compound 3 (12 mg).

The OE $(8.42 \mathrm{~g})$ was partitioned by column chromatography (CC) on $336 \mathrm{~g}$ of silica gel (70-230 mesh, Merck) using mixtures of $\mathrm{n}$-hexane: $\mathrm{CH}_{2} \mathrm{Cl}_{2}: \mathrm{MeOH}$ as eluent. Elution was started with $100 \%$ n-hexane; the polarity of the eluent was then increased by the addition of increasing amounts of $\mathrm{CH}_{2} \mathrm{Cl}_{2}$ (to $100 \%$ ) and then $\mathrm{MeOH}$. This yielded 28 primary fractions (OE1-OE28). Fraction OE11 (1.0 g) was subjected to silica gel CC $(40 \mathrm{~g})$ using mixtures of $\mathrm{n}$ hexane: $\mathrm{CH}_{2} \mathrm{Cl}_{2}: \mathrm{MeOH}$ as eluent. Elution was started with $100 \% \mathrm{n}$-hexane, and the polarity of the eluent was increased by the gradual addition of $\mathrm{CH}_{2} \mathrm{Cl}_{2}$ (to 100\%) and then $\mathrm{MeOH}$. This resulted in 10 subfractions (OE11.1-OE11.10); OE11.10 (40 mg) yielded a mixture of 4 and 5. OE16 (279 mg) was processed with CC on silica gel (11 g) using mixtures of EtOAc:MeOH as eluent, starting with $100 \%$ EtOAc and increasing the polarity with $\mathrm{MeOH}$ to $100 \%$; this process yielded 9 subfractions (OE16.1-OE16.9). Preparative TLC of OE16.4 (32 mg eluted with n-hexane:EtOAc, 1:9) and OE17 (76 mg eluted with $\mathrm{n}$-hexane:EtOAc; $1: 9$ ) resulted in the isolation of $6(10 \mathrm{mg})$ and $7(15 \mathrm{mg})$, respectively. Fraction OE19 (347 mg) was subjected to CC on silica gel (15 g); elution was started with $100 \%$ EtOAc, and the polarity of the solvent was increased by the addition of methanol up to $100 \%$; using this process, it was possible to isolate compound
8 (18 mg). Preparative TLC (EtOAc: $\mathrm{MeOH}: \mathrm{H}_{2} \mathrm{O}, 8: 1.5: 0.5$ ) of fraction OE22 (97 $\mathrm{mg})$ resulted in the isolation of 9 (12 mg). Finally, EO23 (122 mg) and EO24 (434 mg) were dissolved in methanol, and compounds $2(25 \mathrm{mg})$ and $\mathbf{1}$ $(10 \mathrm{mg})$, respectively, were obtained as precipitates.

2.6. Identification of the Isolated Compounds in the Chromatographic Profile of WE. Isolated compounds (1-9) and extracts prepared from $B$. karatas (WE and OE) were injected into the HPLC for identification of the compounds in the chromatographic profiles. Peak assignments were made on the basis of the previously developed structure-diagnostic and supported by examination of the UV spectrum and relative retention time using OpenLAB LC 1260 chromatography software.

\section{Results}

3.1. Ethnobotanical Results. In the field, we confirmed the previous finding that the healer "Isabel Escalante" and the diabetic patients of the town of Tamala use a decoction of $30 \mathrm{~g}$ of the dry leaves of $B$. karatas boiled in 1 liter of water. The infusion is cooled and drunk over the day as "Agua de Uso". The common name of the plant in this region is "timbiriche". Alternatively, the juice of the fruit is used.

3.2. Phytochemical Analysis. The chromatographic profiles were developed to monitor the major components of WE and $\mathrm{OE}$ from B. karatas and to make it possible to isolate and identify them by means of various conventional chromatographic and spectroscopic techniques. The analysis led to the isolation of compounds 1-9, which were identified based on comparison of their ${ }^{1} \mathrm{H}$ and ${ }^{13} \mathrm{C}$ NMR spectral data, including data obtained in 2D experiments (COSY, HSQC, HMBC, NOESY, TOCSY) and their mass spectral data, with those of previously described compounds. The major compounds were identified as a steroidal glycoside, $[\beta$-sitosterol-3-O- $\beta$-D-glucopyranoside (1)] [9], and a phenolic acid, [ $\rho$-coumaric acid, (3)] [10]; both compounds were isolated from the traditional extract (WE). The flavonoid [cirsiliol $4^{\prime}-\mathrm{O}-\beta$-D-glucopyranoside (2)] [11] from WE, along with two steroidal aglycones [stigmasterol (4) and $\beta$-sitosterol (5)] [12], two phenylpropanoid glycerols [1-O-feruloyl-3-O- $\rho$-coumaroylglycerol (6) and 1-O- $\rho$-coumaroyl-3-O-caffeoylglycerol (8)] [13], a phenylpropanoid glycoside $[\beta-\mathrm{D}$-(1-O-acetyl-3,6-O-transdiferuloyl)fructofuranosyl- $\alpha-\mathrm{D}-2^{\prime}, 4^{\prime}, 6^{\prime}-\mathrm{O}$ triacetylglucopyranoside (7)] [14], and 2-propyl-D-glucopyranoside (9) [15] from $\mathrm{OE}$ were some of the minor compounds, Figure 1. The complete spectroscopic data of the isolated compounds are presented below.

$\beta$-Sitosterol-3-O- $\beta$-D-glucopyranoside, (1). Colorless powder; FAB-MS m/z $577[\mathrm{M}+\mathrm{H}]^{+}$for $\mathrm{C}_{35} \mathrm{H}_{60} \mathrm{O}_{6}$; EI-MS m/z 414 [M$\left.\mathrm{C}_{6} \mathrm{H}_{10} \mathrm{O}_{5}\right]^{+} .{ }^{1} \mathrm{H}$ NMR (400 MHz, Pyridine- $\left.d_{5}\right) \delta 5.36(1 \mathrm{H}, \mathrm{t}$, $J=2.6 \mathrm{~Hz}, \mathrm{H}-6), 5.07\left(1 \mathrm{H}, \mathrm{d}, J=7.8 \mathrm{~Hz}, \mathrm{H}-1^{\prime}\right), 4.58(1 \mathrm{H}, \mathrm{dd}, J$ $\left.=11.8,2.5 \mathrm{~Hz}, \mathrm{H}-6^{\prime}\right), 4.43\left(1 \mathrm{H}, \mathrm{dd}, J=11.8,5.1 \mathrm{~Hz}, \mathrm{H}-6^{\prime}\right), 4.30$ $\left(2 \mathrm{H}, \mathrm{m}, \mathrm{H}-3^{\prime}\right.$ and $\left.\mathrm{H}-4^{\prime}\right), 4.08\left(1 \mathrm{H}, \mathrm{t}, J=8.2 \mathrm{~Hz}, \mathrm{H}-2^{\prime}\right), 3.99$ $\left(2 \mathrm{H}, \mathrm{m}, \mathrm{H}-3\right.$ and $\left.\mathrm{H}-5^{\prime}\right), 2.75(1 \mathrm{H}, \mathrm{ddd}, J=13.5,4.8,2.2 \mathrm{~Hz}$, H-12), 2.50 (1H, m, H-12), 2.15 (2H, m, H-2), 1.99 (1H, dd, $J=$ 12.6, $3.5 \mathrm{~Hz}, \mathrm{H}-4), 1.86(1 \mathrm{H}, \mathrm{m}, \mathrm{H}-4), 1.72(1 \mathrm{H}, \mathrm{dtd}, J=18.0,7.9$, 
<smiles>CCC(CC[C@H](C)C1CCC2C3CC=C4C[C@@H](O[C@@H]5O[C@H](CO)[C@@H](O)[C@H](O)[C@H]5O)CC[C@]4(C)C3CC[C@]21C)C(C)C</smiles><smiles>COc1cc2oc(-c3ccc(O[C@@H]4O[C@H](CO)[C@@H](O)[C@H](O)[C@H]4O)c(O)c3)cc(=O)c2c(O)c1OC</smiles><smiles>CCC(/C=C/[C@H](C)C1CCC2C3CC=C4C[C@@H](O)CC[C@]4(C)C3CC[C@@]21C)C(C)C</smiles><smiles>CCC(CC[C@@H](C)C1CCC2C3CC=C4C[C@@H](O)CC[C@]4(C)C3CC[C@@]21C)C(C)C</smiles><smiles>CC(C)O[C@H]1O[C@H](CO)[C@@H](O)[C@H](O)[C@H]1O</smiles>

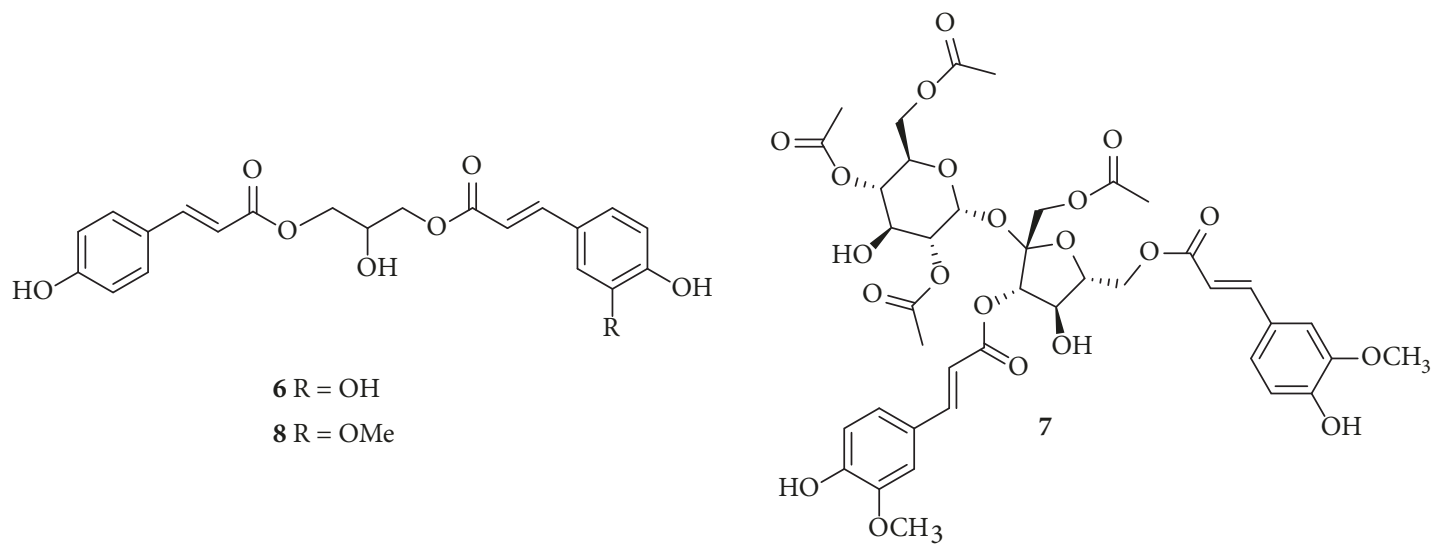

FIGURE 1: Isolated compounds from Bromelia karatas: $\beta$-Sitosterol-3-O- $\beta$-D-glucopyranoside, (1), Cirsiliol $4^{\prime}$-O-glucoside (2), $\rho$ Coumaric acid, (3), Stigmasterol (4), $\beta$-Sitosterol (5), 1-O-Feruloyl-3-O-p-coumaroylglycerol (6), $\beta$-D-(1-O-Acetyl-3,6-O-trans-diferuloyl)fructofuranosyl- $\alpha$-D-2 $4^{\prime}, 6^{\prime}$.-O- triacetylglucopyranoside (7), 1-O-p-Coumaroyl-3-O-caffeoylglycerol (8), 2-Propyl- $\beta$-glucopyranoside (9).

6.9, 3.8 Hz, H-1), $1.68(1 \mathrm{H}, \mathrm{m}, \mathrm{H}-25), 1.41(1 \mathrm{H}, \mathrm{ddt}, J=15.8,9.8$, $4.3 \mathrm{~Hz}, \mathrm{H}-20), 1.36(1 \mathrm{H}, \mathrm{m}, \mathrm{H}-8), 1.10(1 \mathrm{H}, \mathrm{m}, \mathrm{H}-17), 1.02(1 \mathrm{H}$, $\mathrm{m}, \mathrm{H}-24), 1.00(3 \mathrm{H}, \mathrm{d}, J=6.5 \mathrm{~Hz}, \mathrm{H}-21), 0.95(3 \mathrm{H}, \mathrm{s}, \mathrm{H}-19)$, $0.95(1 \mathrm{H}, \mathrm{m}, \mathrm{H}-14), 0.92(3 \mathrm{H}, \mathrm{d}, J=7.3 \mathrm{~Hz}, \mathrm{H}-26) 0.89(3 \mathrm{H}, \mathrm{t}, J$ $=7.4 \mathrm{~Hz}, \mathrm{H}-29), 0.88(3 \mathrm{H}, \mathrm{d}, J=6.9 \mathrm{~Hz}, \mathrm{H}-27), 0.67(3 \mathrm{H}, \mathrm{s}, \mathrm{H}-$ 18). ${ }^{13} \mathrm{C}$ NMR (100 MHz, Pyridine- $\left.d_{5}\right) \delta: 140.91$ (C-5), 121.92 (C-6), $102.57\left(\mathrm{C}-1^{\prime}\right), 78.59(\mathrm{C}-3), 78.47\left(\mathrm{C}-3^{\prime}\right), 78.12\left(\mathrm{C}-5^{\prime}\right)$ $75.30\left(\mathrm{C}-2^{\prime}\right), 71.68\left(\mathrm{C}-4^{\prime}\right), 62.83\left(\mathrm{C}-6^{\prime}\right), 56.84(\mathrm{C}-14), 56.25$ (C-17), 50.35 (C-9), 46.05 (C-24), 42.49 (C-13), 39.96 (C-4), 39.34 (C-12), 37.49 (C-1), 36.93 (C-10), 36.40 (C-20), 34.21 (C22), 32.19 (C-7), 32.06 (C-8), 30.26 (C-2), 29.47 (C-25), 28.56 (C-16), 26.39 (C-23), 24.52 (C-15), 23.40 (C-28), 21.30 (C-11), 20.00 (C-27), 19.43 (C-19), 19.22 (C-26), 19.03 (C-21), 12.17 (C29), 11.99 (C-18).

Cirsiliol 4'-O-glucoside (2). Yellow prisms; HR-ESIMS ion at $\mathrm{m} / \mathrm{z} 515.1204[\mathrm{M}+\mathrm{Na}]^{+}$for $\mathrm{C}_{23} \mathrm{H}_{24} \mathrm{O}_{12} \cdot{ }^{1} \mathrm{H}$ NMR $(400 \mathrm{MHz}$, Pyridine- $\left.d_{5}\right) \delta 7.96\left(1 \mathrm{H}, \mathrm{d}, J=2.2 \mathrm{~Hz}, \mathrm{H}-2^{\prime}\right), 7.72(1 \mathrm{H}, \mathrm{d}, J=$ $\left.8.6 \mathrm{~Hz}, \mathrm{H}-5^{\prime}\right), 7.52\left(1 \mathrm{H}, \mathrm{dd}, J=8.6,2.3 \mathrm{~Hz}, \mathrm{H}-6^{\prime}\right), 7.03(1 \mathrm{H}, \mathrm{s}$, $\mathrm{H}-3), 6.73(1 \mathrm{H}, \mathrm{s}, \mathrm{H}-8), 5.80\left(1 \mathrm{H}, \mathrm{d}, J=7.7 \mathrm{~Hz}, \mathrm{H}^{\prime \prime}{ }^{\prime \prime}\right), 4.61(1 \mathrm{H}$, $\left.\mathrm{dd}, J=12.0,2.1 \mathrm{~Hz}, \mathrm{H}-6_{\mathrm{a}}{ }^{\prime \prime}\right), 4.44(1 \mathrm{H}, \mathrm{dd}, J=12.1,5.3 \mathrm{~Hz}, \mathrm{H}-$ $\left.6_{\mathrm{b}}{ }^{\prime \prime}\right), 4.38\left(1 \mathrm{H}, \mathrm{t}, J=8.9 \mathrm{~Hz}, \mathrm{H}-3^{\prime \prime}\right), 4.34(1 \mathrm{H}, \mathrm{d}, J=8.9 \mathrm{~Hz}, \mathrm{H}-$ $\left.4^{\prime \prime}\right), 4.26\left(1 \mathrm{H}, \mathrm{t}, J=8.9 \mathrm{~Hz}, \mathrm{H}-2^{\prime \prime}\right), 4.18(1 \mathrm{H}, \mathrm{ddd}, J=9.0,5.2$, $\left.2.1 \mathrm{~Hz}, \mathrm{H}-5^{\prime \prime}\right), 4.02\left(3 \mathrm{H}, \mathrm{s}, \mathrm{OCH}_{3}-6\right), 3.91\left(\mathrm{~s}, \mathrm{OCH}_{3}-7\right) .{ }^{13} \mathrm{C}$ NMR (100 MHz, Pyridine- $d_{5}$ ) $\delta 183.55$ (C-4), 164.76 (C-2), 159.83 (C-7), 154.02 (C-9), 153.91 (C-5), 150.54 (C-4'), 149.00 $\left(\mathrm{C}-3^{\prime}\right), 133.57(\mathrm{C}-6), 126.89\left(\mathrm{C}-1^{\prime}\right), 119.7\left(\mathrm{C}-6^{\prime}\right), 118.25\left(\mathrm{C}-5^{\prime}\right)$, 115.83 (C-2' $), 106.87$ (C-10), 105.37 (C-3), $103.54\left(\mathrm{C}-1^{\prime \prime}\right), 91.97$ $(\mathrm{C}-8), 79.68\left(\mathrm{C}-5^{\prime \prime}\right), 78.91\left(\mathrm{C}-3^{\prime \prime}\right), 75.18\left(\mathrm{C}-2^{\prime \prime}\right), 71.57\left(\mathrm{C}-4^{\prime \prime}\right)$, $62.69\left(\mathrm{C}-6^{\prime \prime}\right), 60.96\left(6-\mathrm{OCH}_{3}\right), 56.84\left(7-\mathrm{OCH}_{3}\right)$.

$\rho$-Coumaric Acid, (3). Colorless amorphous powder; ESI-MS ion at $\mathrm{m} / \mathrm{z} 163[\mathrm{M}-\mathrm{H}]^{-}$for $\mathrm{C}_{9} \mathrm{H}_{8} \mathrm{O}_{3} \cdot{ }^{1} \mathrm{H} \mathrm{NMR}(400 \mathrm{MHz}$, $\left.\mathrm{CD}_{3} \mathrm{OD}\right) \delta: 7.50(1 \mathrm{H}, \mathrm{d}, J=15.7 \mathrm{~Hz}, \mathrm{H}-7), 7.41(2 \mathrm{H}, \mathrm{d}, J=$ $8.6 \mathrm{~Hz}, \mathrm{H}-2, \mathrm{H}-6), 6.78(2 \mathrm{H}, \mathrm{d}, J=8.6 \mathrm{~Hz}, \mathrm{H}-3, \mathrm{H}-5), 6.33(1 \mathrm{H}$, $\mathrm{d}, J=15.8 \mathrm{~Hz}, \mathrm{H}-8) .{ }^{13} \mathrm{C}$ NMR $\left(100 \mathrm{MHz}, \mathrm{CD}_{3} \mathrm{OD}\right) \delta 175.20$ (C-9), 158.42 (C-4), 144.18 (C-7), 130.54 (C-2, C-6), 128.01 (C1), 116.66 (C-3, C-5), 115.76 (C-7).

Stigmasterol (4). White powder; ESI-MS ion at $\mathrm{m} / \mathrm{z} 413$ $[\mathrm{M}+\mathrm{H}]^{+}$for $\mathrm{C}_{29} \mathrm{H}_{48} \mathrm{O} .{ }^{1} \mathrm{H}$ NMR $\left(500 \mathrm{MHz}, \mathrm{CDCl}_{3}\right) \delta: 5.35$ $(1 \mathrm{H}, \mathrm{m}, \mathrm{H}-6), 5.15$ (1H, dd, $J=15.2,8.0 \mathrm{~Hz}, \mathrm{H}-21), 5.02(1 \mathrm{H}, \mathrm{dd}$, $J=15.2,8.5 \mathrm{~Hz}, \mathrm{H}-20), 3.52(1 \mathrm{H}, \mathrm{tdd}, J=10.9,5.5,4.1 \mathrm{~Hz}, \mathrm{H}-$ 3), 1.01 (3H, s, H-29), $0.92(3 \mathrm{H}, \mathrm{d}, J=6.6 \mathrm{~Hz}, \mathrm{H}-19), 0.84(3 \mathrm{H}$, t, $J=7.6 \mathrm{~Hz}, \mathrm{H}-24), 0.81(3 \mathrm{H}, \mathrm{d}, J=6.8 \mathrm{~Hz}, \mathrm{H}-26), 0.80(3 \mathrm{H}$, d, $J=6.8 \mathrm{~Hz}, \mathrm{H}-27), 0.68$ (3H, s, H-28). ${ }^{13} \mathrm{C}$ NMR $(125 \mathrm{MHz}$, 
$\left.\mathrm{CDCl}_{3}\right) \delta 140.92(\mathrm{C}-5), 138.5(\mathrm{C}-22), 129.4(\mathrm{C}-23), 121.86(\mathrm{C}-$ 6), 71.97 (C-3), 57.03 (C-14), 56.13 (C-17), 51.39 (C-24), 50.31 (C-9), 42.48 (C-4), 42.38 (C-13), 40.64 (C-20), 39.85 (C-12), 37.42 (C-1), 36.67 (C-10), 32.08 (C-7, C-8, C-25), 31.84 (C-2), 29.01 (C-16), 24.46 (C-15), 25.56 (C-28), 21.37 (C-11), 20.36 (C26), 19.56 (C-27), 18.94 (C-21), 18.94 (C-19), 12.10 (C-29), 12.02 (C-18).

$\beta$-Sitosterol (5). White powder; ESI-MS ion at $\mathrm{m} / \mathrm{z} 415$ $[\mathrm{M}+\mathrm{H}]^{+}$for $\mathrm{C}_{29} \mathrm{H}_{50} \mathrm{O} .{ }^{1} \mathrm{H}$ NMR $\left(500 \mathrm{MHz}, \mathrm{CDCl}_{3}\right) \delta 5.35$ $(1 \mathrm{H}, \mathrm{d}, J=5.3 \mathrm{~Hz}, \mathrm{H}-5), 3.52(1 \mathrm{H}, \mathrm{tdd}, J=10.9,5.5,4.1 \mathrm{~Hz}, \mathrm{H}-$ 3), 1.01 (3H, s, H-29), $0.92(3 \mathrm{H}, \mathrm{d}, J=6.6 \mathrm{~Hz}, \mathrm{H}-19), 0.83(3 \mathrm{H}$, d, $J=6.8 \mathrm{~Hz}, \mathrm{H}-24), 0.81(3 \mathrm{H}, \mathrm{d}, J=6.8 \mathrm{~Hz}, \mathrm{H}-26), 0.69(3 \mathrm{H}$, s, H-28). ${ }^{13} \mathrm{C} \mathrm{NMR}\left(125 \mathrm{MHz}, \mathrm{CDCl}_{3}\right.$ ) $\delta 140.92$ (C-5), 121.86 (C-6), 71.97 (C-3), 57.03 (C-14), 56.13 (C-17), 50.31 (C-9), 46.01 (C-24), 42.48 (C-13), 42.38 (C-4), 39.85 (C-12), 37.42 (C-1), 36.67 (C-10), 36.31 (C-20), 34.12 (C-22), 32.08 (C-2), 31.84 (C7, C-8), 29.33 (C-25), 28.41 (C-16), 26.26 (C-23), 24.46 (C-15), 23.24 (C-28), 21.37 (C-11), 19.98 (C-26), 19.14 (C-27), 19.13 (C19), 18.94 (C-21), 12.21 (C-29), 12.02 (C-18).

1-O-Feruloyl-3-O-p-coumaroylglycerol (6). Colorless oil; ESIMS ion at $\mathrm{m} / \mathrm{z} 437.4[\mathrm{M}+\mathrm{Na}]^{+}$for $\mathrm{C}_{22} \mathrm{H}_{22} \mathrm{O}_{8} \cdot{ }^{1} \mathrm{H} \mathrm{NMR}$ $\left(400 \mathrm{MHz}, \mathrm{CD}_{3} \mathrm{OD}\right) \delta 7.66\left(2 \mathrm{H}, \mathrm{d}, J=16.0 \mathrm{~Hz}, \mathrm{H}-7^{\prime}, \mathrm{H}-7^{\prime \prime}\right)$, $7.44\left(2 \mathrm{H}, \mathrm{d}, J=8.7 \mathrm{~Hz}, \mathrm{H}-2^{\prime}, \mathrm{H}-6^{\prime}\right), 7.18(1 \mathrm{H}, \mathrm{d}, J=2.0 \mathrm{~Hz}, \mathrm{H}-$ $\left.2^{\prime \prime}\right), 7.07\left(1 \mathrm{H}, \mathrm{dd}, J=8.3,2.1 \mathrm{~Hz}, \mathrm{H}-6^{\prime \prime}\right), 6.80(2 \mathrm{H}, \mathrm{d}, J=8.2 \mathrm{~Hz}$, H-3', H-5') $6.79\left(1 \mathrm{H}, \mathrm{d}, J=8.6 \mathrm{~Hz}, \mathrm{H}-5^{\prime \prime}\right), 6.39(1 \mathrm{H}, \mathrm{d}, J=$ $\left.14.2 \mathrm{~Hz}, \mathrm{H}-8^{\prime \prime}\right), 6.35\left(1 \mathrm{H}, \mathrm{d}, J=14.3 \mathrm{~Hz}, \mathrm{H}-8^{\prime}\right), 4.28(4 \mathrm{H}, \mathrm{dd}, J$ $=5.3,1.9 \mathrm{~Hz}, \mathrm{H}-1, \mathrm{H}-3), 4.16(1 \mathrm{H}, \mathrm{m}, \mathrm{H}-2), 3.87\left(3 \mathrm{H}, \mathrm{s}, \mathrm{OCH}_{3}-\right.$ $\left.3^{\prime \prime}\right) .{ }^{13} \mathrm{CNMR}\left(100 \mathrm{MHz}, \mathrm{CD}_{3} \mathrm{OD}\right) \delta 169.01\left(\mathrm{C}-9^{\prime \prime}\right), 168.99(\mathrm{C}-$ $\left.9^{\prime}\right), 161.34\left(\mathrm{C}-4^{\prime}\right), 150.71\left(\mathrm{C}-3^{\prime \prime}\right), 149.38\left(\mathrm{C}-4^{\prime \prime}\right), 147.25\left(\mathrm{C}-7^{\prime \prime}\right)$, $146.97\left(\mathrm{C}-7^{\prime}\right), 131.21\left(\mathrm{C}-2^{\prime}, \mathrm{C}-6^{\prime}\right), 127.66\left(\mathrm{C}-1^{\prime \prime}\right), 127.11\left(\mathrm{C}-1^{\prime}\right)$, $124.19\left(\mathrm{C}-6^{\prime \prime}\right), 116.84\left(\mathrm{C}-3^{\prime}, \mathrm{C}-5^{\prime}\right), 116.48\left(\mathrm{C}-5^{\prime \prime}\right), 115.10\left(\mathrm{C}-8^{\prime \prime}\right)$, $114.80\left(\mathrm{C}-8^{\prime}\right), 111.75\left(\mathrm{C}-2^{\prime \prime}\right), 68.61(\mathrm{C}-2), 66.39(\mathrm{C}-3), 66.37(\mathrm{C}-$ 1), $56.44\left(\mathrm{OCH}_{3}-3^{\prime \prime}\right)$.

$\beta$-D-(1-O-Acetyl-3,6-O-trans-diferuloyl)-fructofuranosyl$\alpha-D-2^{\prime}, 4^{\prime}, 6^{\prime} .-O$ - triacetylglucopyranoside (7). Colorless amorphous solid, ESI-MS ion at $\mathrm{m} / \mathrm{z} 885.2[\mathrm{M}+\mathrm{Na}]^{+}$for $\mathrm{C}_{40} \mathrm{H}_{46} \mathrm{O}_{21} \cdot{ }^{1} \mathrm{H}$ NMR $\left(500 \mathrm{MHz}, \mathrm{CD}_{3} \mathrm{OD}\right) \delta 7.72(1 \mathrm{H}, \mathrm{d}, J=$ $\left.15.9 \mathrm{~Hz}, \mathrm{H}-7^{\prime \prime}\right), 7.68\left(1 \mathrm{H}, \mathrm{d}, J=15.9 \mathrm{~Hz}, \mathrm{H}-7^{\prime \prime \prime}\right), 7.28(2 \mathrm{H}, \mathrm{d}, J$ $\left.=2.0 \mathrm{~Hz}, \mathrm{H}-2^{\prime \prime}\right), 7.22\left(2 \mathrm{H}, \mathrm{d}, J=2.0 \mathrm{~Hz}, \mathrm{H}-2^{\prime \prime \prime}\right), 7.13(1 \mathrm{H}, \mathrm{dd}, J$ $\left.=8.5,2.01 \mathrm{~Hz}, \mathrm{H}-6^{\prime \prime}\right), 7.11\left(1 \mathrm{H}, \mathrm{dd}, J=8.5,1.9 \mathrm{~Hz}, \mathrm{H}-6^{\prime \prime \prime}\right), 6.83$ $\left(2 \mathrm{H}, \mathrm{d}, J=8.2 \mathrm{~Hz}, \mathrm{H}-5^{\prime \prime}, \mathrm{H}-5^{\prime \prime \prime}\right), 6.46(1 \mathrm{H}, \mathrm{d}, J=14.42 \mathrm{~Hz}$, $\left.\mathrm{H}-8^{\prime \prime}\right), 6.43\left(1 \mathrm{H}, \mathrm{d}, J=14.4 \mathrm{~Hz}, \mathrm{H}-8^{\prime \prime \prime}\right), 5.70(1 \mathrm{H}, \mathrm{d}, J=3.8 \mathrm{~Hz}$, $\left.\mathrm{H}-1^{\prime}\right), 5.39(1 \mathrm{H}, \mathrm{d}, J=7.7 \mathrm{~Hz}, \mathrm{H}-3), 4.80(1 \mathrm{H}, \mathrm{d}, J=10.2 \mathrm{~Hz}$, $\left.\mathrm{H}-4^{\prime}\right), 4.68\left(1 \mathrm{H}, \mathrm{dd}, J=10.2,3.8 \mathrm{~Hz}, \mathrm{H}-2^{\prime}\right), 4.50(1 \mathrm{H}, \mathrm{dd}, J=$ 12.1, 3.27 Hz, H-6 $) 4.45\left(1 \mathrm{H}, \mathrm{m}, \mathrm{H}-6_{\mathrm{b}}\right), 4.46(1 \mathrm{H}, \mathrm{t}, J=7.9 \mathrm{~Hz}$, $\mathrm{H}-4), 4.26,\left(\mathrm{~d}, J=11.5 \mathrm{~Hz}, \mathrm{H}-1_{\mathrm{a}}\right), 4.24\left(1 \mathrm{H}, \mathrm{m}, \mathrm{H}-5^{\prime}\right), 4.21$ $\left(\mathrm{d} \mathrm{br}, J=11.4 \mathrm{~Hz}, \mathrm{H}-6^{\prime}{ }_{\mathrm{a}}\right), 4.10\left(1 \mathrm{H}, \mathrm{dd}, J=11.6,3.5 \mathrm{~Hz}, \mathrm{H}-6_{\mathrm{b}}{ }_{\mathrm{b}}\right)$ $4.09\left(1 \mathrm{H}, \mathrm{d}, J=11.5 \mathrm{~Hz}, \mathrm{H}-1_{\mathrm{b}}\right), 3.91\left(3 \mathrm{H}, \mathrm{s}, \mathrm{OCH}_{3}-3^{\prime \prime \prime}\right), 3.91$ $\left(3 \mathrm{H}, \mathrm{s}, \mathrm{OCH}_{3}-3^{\prime \prime}\right), 3.88\left(1 \mathrm{H}, \mathrm{d}, J=7.6 \mathrm{~Hz}, \mathrm{H}-3^{\prime}\right), 2.13(3 \mathrm{H}, \mathrm{s}$, OAc-1), 2.10 (3H, s, OAc- $\left.{ }^{\prime}\right), 2.03\left(3 \mathrm{H}, \mathrm{s}, \mathrm{OAc}-6^{\prime}\right), 1.87(3 \mathrm{H}$, $\left.\mathrm{s}, \mathrm{OAc}-4^{\prime}\right) .{ }^{13} \mathrm{C} \mathrm{NMR}\left(125 \mathrm{MHz}, \mathrm{CD}_{3} \mathrm{OD}\right) \delta 172.60\left(\mathrm{OAc}^{\prime}{ }^{\prime}\right)$, $172.14\left(\mathrm{OAc}^{\prime}\right), 172.02$ (OAc-1), $171.72\left(\mathrm{OAc}-4^{\prime}\right), 168.77$ $\left(\mathrm{C}-9^{\prime \prime \prime}\right), 167.98\left(\mathrm{C}-9^{\prime \prime}\right), 151.12\left(\mathrm{C}-4^{\prime \prime}\right), 150.76\left(\mathrm{C}-4^{\prime \prime \prime}\right), 149.51$ $\left(\mathrm{C}-3^{\prime \prime}\right), 149.43\left(\mathrm{C}-3^{\prime \prime \prime}\right), 148.23\left(\mathrm{C}-7^{\prime \prime}\right), 147.25\left(\mathrm{C}-7^{\prime \prime \prime}\right), 127.74$ $\left(\mathrm{C}-1^{\prime \prime}\right), 127.50\left(\mathrm{C}-1^{\prime \prime \prime}\right), 124.62\left(\mathrm{C}-6^{\prime \prime}\right), 124.31\left(\mathrm{C}-6^{\prime \prime \prime}\right), 116.57$ $\left(\mathrm{C}-5^{\prime \prime}\right), 116.52\left(\mathrm{C}-5^{\prime \prime \prime}\right), 115.15\left(\mathrm{C}-8^{\prime \prime \prime}\right), 114.30\left(\mathrm{C}-8^{\prime \prime}\right), 111.83$
$\left(\mathrm{C}-2^{\prime \prime}\right), 111.68\left(\mathrm{C}-2^{\prime \prime \prime}\right), 103.91(\mathrm{C}-2), 90.46\left(\mathrm{C}-1^{\prime}\right), 81.52(\mathrm{C}-5)$, 79.55 (C-3), 73.95 (C-2'), 73.79 (C-4), $72.25\left(\mathrm{C}-4^{\prime}\right), 70.06$

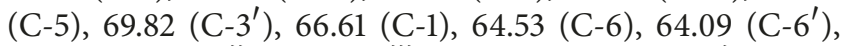

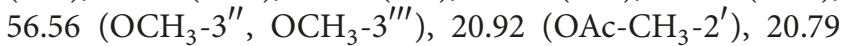

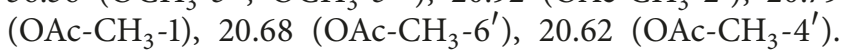
Assignments of the acetate and feruloyl units at positions $1,2^{\prime}, 4^{\prime}, 6^{\prime}$ (for acetyl) and 3, 6 (for feruloyl), respectively, in the sucrose molecule were corroborated by the HMBC two-dimensional spectrum; this showed a correlation between methine and methylene protons at $1,2^{\prime}, 4^{\prime}$ and $6^{\prime}$ of the sucrose with methyl protons and the carbonyl carbon of the acetyl groups $\left({ }^{1} \mathrm{H} /{ }^{13} \mathrm{C} /{ }^{1} \mathrm{H}\right.$ : 4.26, 4.09/172.02/2.13 for $1 ; 4.68 / 172.14 / 2.10$ for $2^{\prime} ; 4.80 / 171.72 / 1.87$ for $4^{\prime} ; 4.21$, $4.10 / 172.60 / 2.03$ for $6^{\prime}$ ) and between methine and methylene protons at 3 and 6 of the fructose with the protons at $8^{\prime \prime}$ and $8^{\prime \prime \prime}$ of the feruloyl moiety and the carbonyl carbons at $9^{\prime \prime}$ and $9^{\prime \prime \prime}\left({ }^{1} \mathrm{H} /{ }^{13} \mathrm{C} /{ }^{1} \mathrm{H}\right.$ : 6.46/167.98/5.39 for C-3 feruloyl; $6.43 / 168.77 / 4.50,4.45$ for C-3 feruloyl).

1-O-p-Coumaroyl-3-O-caffeoylglycerol (8). Colorless oil; ESIMS ion at $\mathrm{m} / \mathrm{z} 399.8[\mathrm{M}-\mathrm{H}]^{-}$for $\mathrm{C}_{21} \mathrm{H}_{20} \mathrm{O}_{8} \cdot{ }^{1} \mathrm{H} \mathrm{NMR}$ $\left(400 \mathrm{MHz}, \mathrm{CD}_{3} \mathrm{OD}\right) \delta 7.66\left(1 \mathrm{H}, \mathrm{d}, J=16.0 \mathrm{~Hz}, \mathrm{H}-7^{\prime}\right), 7.60(1 \mathrm{H}$, $\left.\mathrm{d}, J=15.9 \mathrm{~Hz}, \mathrm{H}-7^{\prime \prime}\right), 7.44\left(2 \mathrm{H}, \mathrm{dd}, J=8.9,2.3 \mathrm{~Hz}, \mathrm{H}-2^{\prime}, \mathrm{H}-6^{\prime}\right)$, $7.05\left(1 \mathrm{H}, \mathrm{d}, J=2.1 \mathrm{~Hz}, \mathrm{H}-2^{\prime \prime}\right), 6.94(1 \mathrm{H}, \mathrm{dd}, J=8.3,2.0 \mathrm{~Hz}, \mathrm{H}-$ $\left.6^{\prime \prime}\right), 6.79\left(2 \mathrm{H}, \mathrm{dd}, J=9.1,2.5 \mathrm{~Hz}, \mathrm{H} 3^{\prime}, \mathrm{H}-5^{\prime}\right), 6.77(1 \mathrm{H}, \mathrm{d}, J=$ $\left.8.4 \mathrm{~Hz}, \mathrm{H}-5^{\prime \prime}\right), 6.35\left(1 \mathrm{H}, \mathrm{d}, J=15.9 \mathrm{~Hz}, \mathrm{H}-8^{\prime}\right), 6.30(1 \mathrm{H}, \mathrm{d}, J=$ $\left.15.9 \mathrm{~Hz}, \mathrm{H}-8^{\prime \prime}\right), 4.27(4 \mathrm{H}, \mathrm{d}, J=5.4 \mathrm{~Hz}, \mathrm{H}-1, \mathrm{H}-3), 4.16(1 \mathrm{H}, \mathrm{q}$, $J=5.2 \mathrm{~Hz}, \mathrm{H}-2) .{ }^{13} \mathrm{C} \mathrm{NMR}\left(100 \mathrm{MHz}, \mathrm{CD}_{3} \mathrm{OD}\right) \mathrm{d} 169.01(\mathrm{C}-$ $\left.9^{\prime}, \mathrm{C}-9^{\prime \prime}\right), 161.31\left(\mathrm{C}-4^{\prime}\right), 149.65\left(\mathrm{C}-3^{\prime \prime}\right), 147.36\left(\mathrm{C}-7^{\prime \prime}\right), 146.97$ $\left(\mathrm{C}-7^{\prime}\right), 146.81\left(\mathrm{C}-4^{\prime \prime}\right), 131.22\left(\mathrm{C}-2^{\prime}, \mathrm{C}-6^{\prime}\right), 127.70\left(\mathrm{C}-1^{\prime \prime}\right), 127.13$ $\left(\mathrm{C}-1^{\prime}\right), 123.02\left(\mathrm{C}-6^{\prime \prime}\right), 116.83\left(\mathrm{C}-3^{\prime}, \mathrm{C}-5^{\prime}\right), 116.51\left(\mathrm{C}-5^{\prime \prime}\right), 115.20$ $\left(\mathrm{C}-2^{\prime \prime}\right), 114.82\left(\mathrm{C}-8^{\prime}\right), 114.76\left(\mathrm{C}-8^{\prime \prime}\right), 68.65(\mathrm{C}-2), 66.35(\mathrm{C}-1$, C-3).

2-Propyl- $\beta$-glucopyranoside (9). Colorless amorphous solid, ESI-MS ion at $\mathrm{m} / \mathrm{z} 245.2[\mathrm{M}+\mathrm{Na}]^{+}$for $\mathrm{C}_{9} \mathrm{H}_{18} \mathrm{O}_{6} \cdot{ }^{1} \mathrm{H} \mathrm{NMR}$ $\left(400 \mathrm{MHz}, \mathrm{CD}_{3} \mathrm{OD}\right) \delta 4.34(1 \mathrm{H}, \mathrm{d}, J=7.8 \mathrm{~Hz}, \mathrm{H}-1), 4.04(1 \mathrm{H}$, hept, $\left.J=6.1 \mathrm{~Hz}, \mathrm{H}-2^{\prime}\right), 3.85\left(1 \mathrm{H}, \mathrm{dd}, J=11.9,2.0 \mathrm{~Hz}, \mathrm{H}-6_{\mathrm{b}}\right)$, $3.66\left(1 \mathrm{H}, \mathrm{dd}, J=11.9,5.4 \mathrm{~Hz}, \mathrm{H}-6_{\mathrm{a}}\right), 3.35(1 \mathrm{H}, \mathrm{m}, \mathrm{H}-3), 3.27$ $(1 \mathrm{H}, \mathrm{m}, \mathrm{H}-4), 3.25(1 \mathrm{H}, \mathrm{m}, \mathrm{H}-5), 3.14(1 \mathrm{H}, \mathrm{dd}, J=9.1,7.8 \mathrm{~Hz}$, $\mathrm{H}-2), 1.23\left(3 \mathrm{H}, \mathrm{d}, J=6.2 \mathrm{~Hz}, \mathrm{CH}_{3}-^{\prime}\right), 1.19(3 \mathrm{H}, \mathrm{d}, J=6.1 \mathrm{~Hz}$, $\left.\mathrm{CH}_{3}-3^{\prime}\right) .{ }^{13} \mathrm{C}$ NMR (100 MHz, CD $\left.\mathrm{OD}\right) \delta 102.55$ (C-1), 78.11 (C-3), 77.86 (C-5), 75.14 (C-2), 72.58 (C-2' ), 71.70 (C-4), 62.79 (C-6), $23.81\left(\mathrm{C}-1^{\prime}\right), 22.04\left(\mathrm{C}-3^{\prime}\right)$.

3.3. Chromatographic Profiles. In the HPLC profiles of the WE and the OE of B. karatas (Figure 1), the majority of components showed maximum absorption between 280 and $320 \mathrm{~nm}$. The two chromatograms were qualitatively different; however, the main components of WE $\left[\mathrm{T}_{\mathrm{R}}: 13.3(3), 14.2\right.$ and 21.9 (2) $\mathrm{min}$ ] were also observed in the $\mathrm{OE}\left[\mathrm{T}_{\mathrm{R}}: 13.3(3), 14.2\right.$ and 21.9 (2) $\mathrm{min}$ ] but in different proportion. Compounds 4, 5 , and 9 were not observed in any of the chromatographic profiles, Figure 2.

Compound 1 was not observed as a major compound in the profile of the WE (Figure 2(a)) because it precipitated during the process of sample preparation before the sample was injected into the HPLC equipment; its remnant was 


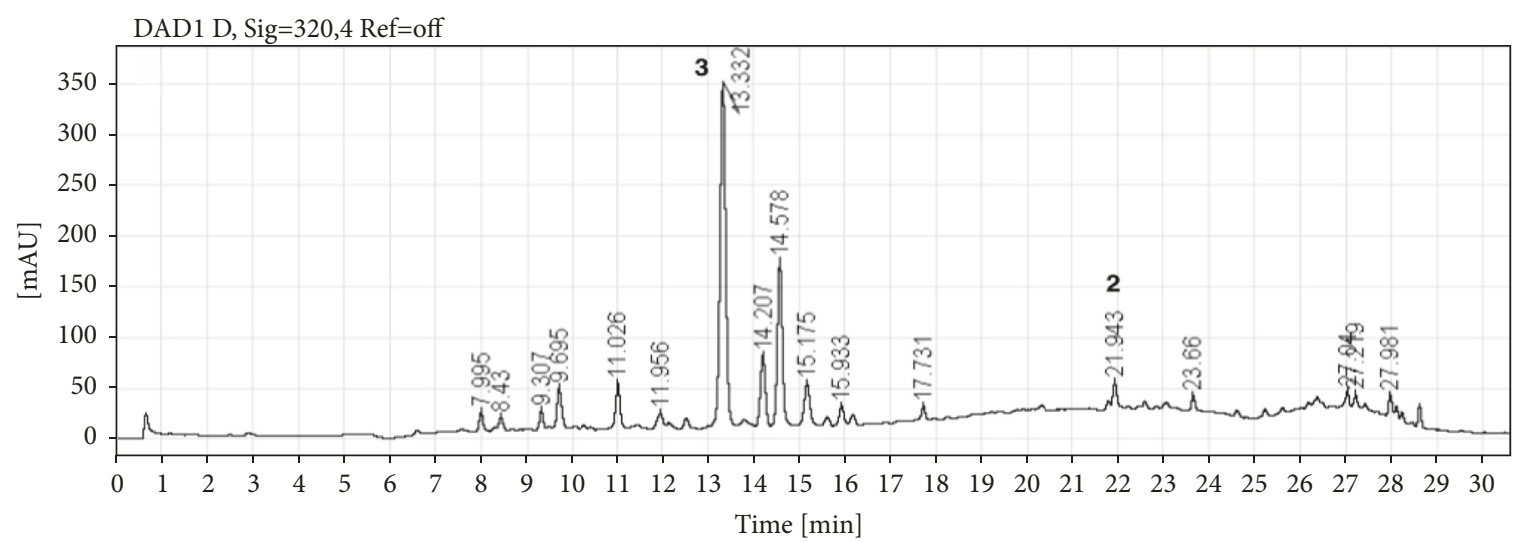

(a)

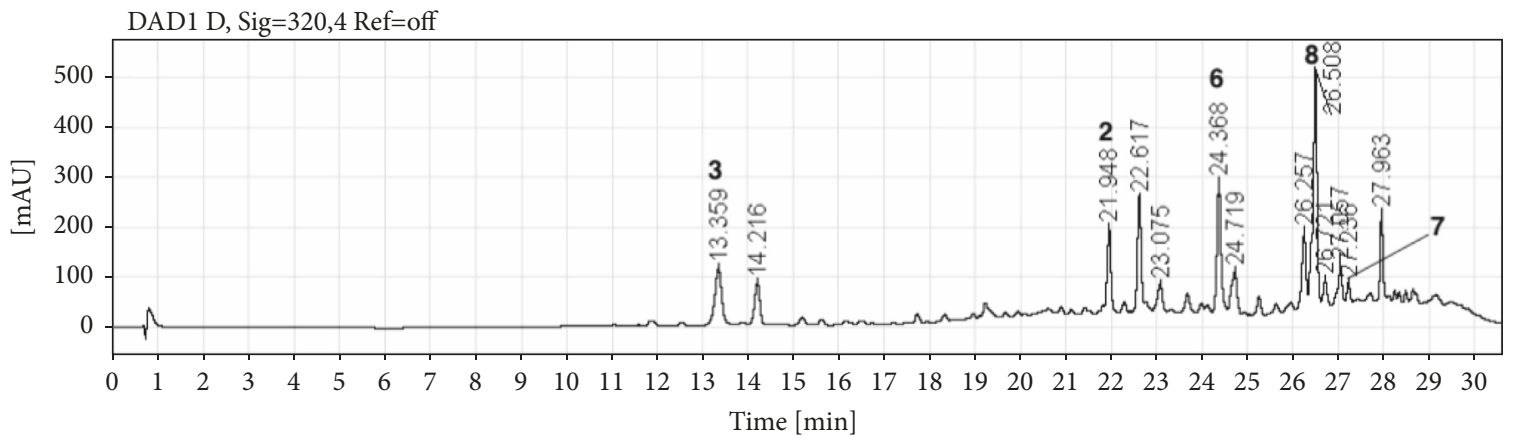

(b)

FIgURE 2: HPLC-DAD profiles of the WE and the OE. (a) Water extract; (b) organic extract.

observed at $254 \mathrm{~nm}$ at a retention time of $30.65 \mathrm{~min}$ (data not shown).

3.4. Hypoglycemic Effect of the Water Extract in a Chronic Trial for 42 Days. After the induction of experimental hyperglycemia, the HC group presented higher glucose values than the NC group throughout the 42-day duration of the study, while in the GB group, a hypoglycemic effect was observed from days 7 to 42; this effect was statistically significant compared with the HG group and with the time 0 values of the GB group. A similar hypoglycemic effect beginning at day 7 and continuing until day 42 was observed after the oral administration of BK-WE, confirming that both the plant extract and the drug glibenclamide exert a chronic hypoglycemic effect (see Table 1).

The levels of glycated hemoglobin were elevated in the HC group compared to the NC, CG, and BK-WE groups. Both glibenclamide and the extract controlled the increase in the values of $\mathrm{HB} 1 \mathrm{Ac}$, but the effect was not statistically significant compared to the HG or to the animals' own time 0 values. The cholesterol values were similar in all the groups (data not shown), and vLDL levels were not modified by the control drug or by the extract (see Table 2).

3.5. Hypoglycemic Effect of the Isolated Compounds. After the induction of experimental hyperglycemia, the hyperglycaemic group (HC) presented higher glucose values than the normoglycemic group (NC). Through the 180 minutes, both groups present a stable glycemia, the NC around $100 \mathrm{mg} / \mathrm{dl}$ and the $\mathrm{HC}$ around $190 \mathrm{mg} / \mathrm{dl}$, with no statistical difference between their own time 0 . However, HC presents higher glucose levels compared to NC. When the hypoglycemic agent glibenclamide $(5 \mathrm{mg} / \mathrm{kg})$ was administrated (GC) a statistically significant hypoglycemic effect from 60 to 180 min compared against the $\mathrm{HC}$ and their own time 0 could be observed. The compound (1) $\beta$-Sitosterol-3-O- $\beta$-Dglucopyranoside exerts a hypoglycemic effect, but it is only statistically significant at $180 \mathrm{~min}$. The compound (2) Cirsiliol $4^{\prime}$-O-glucoside exerts a statistically significant hypoglycemic effect from 60 to $180 \mathrm{~min}$, like glibenclamide. The compound (3) $\rho$-Coumaric acid also produces a hypoglycemic effect since $120 \mathrm{~min}$, but it is only statistically significant at time $180 \mathrm{~min}$ (see Table 3).

\section{Discussion}

The results of the present work support the traditional use of $B$. karatas in the treatment of type 2 diabetes. The extract tested here, which is similar to the traditionally used infusion, possesses a chronic hypoglycemic effect, and the observed effect was sustained throughout a 42 -day period. The plant extract was also able to control the elevation in glycated hemoglobin with no effect on cholesterol or vLDL levels. 
TABLE 1: Chronic hypoglycemic effect of Bromelia karatas on STZ-NA induced diabetic rats.

\begin{tabular}{|c|c|c|c|c|c|c|c|}
\hline Glucose & $(\mathrm{mg} / \mathrm{dl})$ & (mg/dl) & (mg/dl) & $(\mathrm{mg} / \mathrm{dl})$ & (mg/dl) & (mg/dl) & $(\mathrm{mg} / \mathrm{dl})$ \\
\hline Groups & T0 & $\mathrm{T} 7$ & $\mathrm{~T} 14$ & $\mathrm{~T} 21$ & T28 & Т35 & $\mathrm{T} 42$ \\
\hline $\mathrm{NC}$ & $124 \pm 3.2$ & $129 \pm 1.7$ & $124 \pm 2.4$ & $127 \pm 1.1$ & $125 \pm 4.3$ & $118 \pm 6.4$ & $131 \pm 2.7$ \\
\hline $\mathrm{HC}$ & $174 \pm 10.6^{1}$ & $171 \pm 1.5^{1}$ & $172 \pm 9.7^{1}$ & $153 \pm 3.8^{1}$ & $168 \pm 7.3^{1}$ & $162 \pm 3.8^{1}$ & $169 \pm 8.7^{1}$ \\
\hline $\begin{array}{l}\text { CG } \\
5 \mathrm{mg} / \mathrm{kg}\end{array}$ & $180 \pm 2.5^{1}$ & $128 \pm 4.9^{\mathrm{a}, 1}$ & $147 \pm 9.4^{\mathrm{a}, 1}$ & $133 \pm 6.2^{\mathrm{a}, 1}$ & $150 \pm 9^{a, 1}$ & $134 \pm 11.4^{\mathrm{a}, 1}$ & $152 \pm 10.8^{a, 1}$ \\
\hline $\begin{array}{l}\text { Bk-WE } \\
218 \mathrm{mg} / \mathrm{Kg}\end{array}$ & $186 \pm 3.1^{1}$ & $138 \pm 3.3^{\mathrm{a}, 1}$ & $146 \pm 2.8^{\mathrm{a}, 1}$ & $138 \pm 3.1^{\mathrm{a}, 1}$ & $141 \pm 5.1^{a, 1}$ & $132.8 \pm 4.2^{a, 1}$ & $140.2 \pm 7.8^{a, 1}$ \\
\hline
\end{tabular}

The values represent the mean \pm SEM. Superscripted numbers in the same column indicate statistically significant differences compared with the respective control group. Superscripted letters in the same row indicate statistically significant differences compared with time $0 . a, 1(p<0.05)$.

TABLE 2: Chronic hypoglycemic effects of Bromelia karatas on STZ-NA induced diabetic rats.

\begin{tabular}{|c|c|c|c|c|c|c|c|c|}
\hline \multirow[b]{2}{*}{ Groups } & \multicolumn{2}{|c|}{ TO } & \multicolumn{2}{|c|}{ T14 } & \multicolumn{2}{|c|}{ T28 } & \multicolumn{2}{|c|}{ T42 } \\
\hline & $\begin{array}{c}H b A 1 c \\
(\%)\end{array}$ & $\begin{array}{c}v L D L \\
(m g / d l)\end{array}$ & $\begin{array}{c}H b A 1 c \\
(\%)\end{array}$ & $\begin{array}{c}v L D L \\
(m g / d l)\end{array}$ & $\begin{array}{c}H b A 1 c \\
(\%)\end{array}$ & $\begin{array}{c}v L D L \\
(m g / d l)\end{array}$ & $\begin{array}{c}H b A 1 c \\
(\%)\end{array}$ & $\begin{array}{c}v L D L \\
(m g / d l)\end{array}$ \\
\hline $\mathrm{NC}$ & $3.6 \pm 0.1$ & $14.2 \pm .8$ & $3.6 \pm 0.1$ & $15.8 \pm 3$ & $3.5 \pm 0.1$ & $12.6 \pm .8$ & $3.6 \pm 0.1$ & $12.2 \pm 2$ \\
\hline $\mathrm{HC}$ & $3.7 \pm 0.1$ & $10.4 \pm 1$ & $4.1 \pm .2$ & $15 \pm 2.7$ & $4.2 \pm 0.1^{1, \mathrm{a}}$ & $23.8 \pm 3^{1, a}$ & $4.3 \pm 0.1^{1, \mathrm{a}}$ & $22.6 \pm 5^{a}$ \\
\hline $\begin{array}{l}\text { CG } \\
5 \mathrm{mg} / \mathrm{kg}\end{array}$ & $3.8 \pm 0.2$ & $13.8 \pm 2$ & $4.2 \pm 0.2^{1, \mathrm{a}}$ & $17.8 \pm 3$ & $3.9 \pm 0.2$ & $20 \pm 3$ & $3.9 \pm 0.2$ & $23 \pm 3.3^{a}$ \\
\hline $\begin{array}{l}\text { Bk-WE } \\
218 \mathrm{mg} / \mathrm{Kg}\end{array}$ & $3.6 \pm 0.1$ & $20 \pm 2^{1}$ & $4.1 \pm 0.2$ & $21 \pm 4.6$ & $4.0 \pm 0.1$ & $17.2 \pm 3$ & $4.1 \pm 0.1$ & $25 \pm 6$ \\
\hline
\end{tabular}

The values represent the mean \pm SEM. Superscripted letters in the same row indicate statistically significant differences compared with time 0 . Superscripted numbers in the same column indicate statistically significant differences compared with the respective control group. a,1 ( $p<0.0)$. VLDL was calculated using the following VLDL $=0.2 \times \mathrm{TG}$.

The chromatographic profiles and the absorption spectra of the extracts of B. karatas indicated the presence of phenolic compounds and flavonoids. The organic extract was used to increase the chemical profile of the plant, making it possible to isolate several phenolic compounds, including glycosides and glycerides of phenylpropanoids, flavonoids, and phytosterols. 1, 4, and $\mathbf{5}$ are the major phytosterols in higher plants; $\mathbf{2}$ is part of the structural group of polymethoxylated flavones, which are distributed mainly in the Rutaceae and have been shown to have a broad spectrum of biological activity that includes anti-inflammatory, anticancer, and antiatherogenic properties [16]; 3, 6, and $\mathbf{8}$ have been previously described in the family Bromeliaceae [17]; 7 was isolated for the first time from Sparganium stoloniferum [14]; and 9 is not commonly isolated from natural products. Sharma et al. (1989) [18] isolated the isopropyl- $\alpha$-D-glucopyranoside from the coral Sclerophytum capitalis; they assumed that this compound is not an artifact produced during the investigation because, similar to us, they did not use propanol in the extraction and purification process. It should be noted that 1-9 have not been reported previously for $B$. karatas. $\mathbf{1}$ and $\mathbf{3}$ were considered the major compounds in the infusion (WE). Several studies show that saponins regulate blood glucose levels and prevent diabetic complications due to their antioxidant activity [19]; sitosterol-3-O- $\beta$-D-glucopyranoside (1) was shown to have hypoglycemic and antihyperglycemic effects in STZ-NA rats treated with doses of 0.25 and $0.50 \mathrm{mg} / \mathrm{kg}$ for 21 days using glibenclamide as a positive control and to improve biochemical and hematological parameters such as total cholesterol, triglycerides, high-density lipoprotein (HDL), low-density lipoprotein (LDL), blood urea nitrogen, creatinine, red blood cells, platelets, and white blood cells, [20]. On the other hand, Amalam et al. (2016) [21] demonstrated the "antidiabetic" potential of $\rho$-coumaric acid (3) by showing that it exerts a protective role in pancreatic b-cells of diabetic rats by reducing ROS-induced oxidative stress and improving antioxidant status and by providing evidence for the participation of GLUT-2 in controlling glucose homeostasis. A more recent trial showed the powerful antihyperglycemic and antihyperlipidemic efficacy of $\rho$-coumaric acid in STZ-NA rats treated orally with $40 \mathrm{mg} / \mathrm{kg}$ body mass for six weeks; this process may be mediated via modulation of TNF- $\alpha$ and adipocytokine secretion as well as by upregulation of PPAR $\gamma$ mRNA expression [22]. The effect of the WE from Bromelia karatas in this test was similar to that of glibenclamide; consequently, we were able to confirm that $\mathbf{1}$ and $\mathbf{3}$ are the active principles of the plant.

The previously reported hypoglycemic effect of compounds 1 and 3 is here supported by our own data, for $\beta$ Sitosterol-3-O- $\beta$-D-glucopyranoside we observe nearly $80 \%$ of glucose reduction after $180 \mathrm{~min}$, a similar result was previously described in a chronic experiment [20], and also the previous observation about the hypoglycemic effect of $\rho$-Coumaric acid observed by [21] was corroborated here. 
TABLE 3: Acute hypoglycemic effect of the isolated compounds.

\begin{tabular}{|c|c|c|c|c|}
\hline \multirow[b]{2}{*}{ Group/Time (min.) } & \multicolumn{4}{|c|}{ Glucose levels in the maltose curve [mg/dl] } \\
\hline & T0 & T60 & T120 & T180 \\
\hline Normoglycemic control & $106 \pm 5^{b}$ & $114 \pm 9^{\mathrm{b}}$ & $110 \pm 7^{\mathrm{b}}$ & $105 \pm 5^{\mathrm{b}}$ \\
\hline Hyperglycaemic control & $196 \pm 7$ & $191 \pm 7$ & $187 \pm 3$ & $194 \pm 6$ \\
\hline $\begin{array}{l}\text { Hyperglycemic + glibenclamide } \\
5 \mathrm{mg} / \mathrm{kg}\end{array}$ & $192 \pm 8$ & $118 \pm 10^{\mathrm{a}, \mathrm{b}}$ & $107 \pm 7^{\mathrm{a}, \mathrm{b}}$ & $106 \pm 9^{\mathrm{a}, \mathrm{b}}$ \\
\hline$\beta$-Sitosterol-3-O- $\beta$-D-glucopyranoside. $72 \mathrm{mg} / \mathrm{kg}$ & $186 \pm 6$ & $203 \pm 5$ & $172 \pm 2$ & $156 \pm 7^{b}$ \\
\hline $\begin{array}{l}\text { Cirsiliol } 4^{\prime}-O \text {-glucoside } \\
1.8 \mathrm{mg} / \mathrm{kg}\end{array}$ & $194 \pm 5$ & $179 \pm 8^{\mathrm{a}}$ & $160 \pm 10^{\mathrm{a}, \mathrm{b}}$ & $133 \pm 14^{\mathrm{a}, \mathrm{b}}$ \\
\hline $\begin{array}{l}\rho \text {-Coumaric acid } \\
3.63 \mathrm{mg} / \mathrm{kg}\end{array}$ & $187 \pm 8$ & $198 \pm 7$ & $180 \pm 7^{\mathrm{a}}$ & $166 \pm 9^{a}$ \\
\hline
\end{tabular}

The values represent the mean \pm SEM. In the same row: a indicates statistically significant differences compared with time 0 . In the same column: $b$ indicates statistically significant differences compared with the diabetic control group; $\mathrm{p}<0.05, \mathrm{n}=3$.

For the first time, the hypoglycemic effect of Cirsiliol $4^{\prime}-\mathrm{O}-$ glucoside is reported, which exerts better effect with a $30 \%$ reduction in blood sugar levels after $180 \mathrm{~min}$.

This work supports the traditional use of the plant to treat type 2 diabetes and describes the compound Cirsiliol $4^{\prime}-\mathrm{O}-$ glucoside as a novel hypoglycemic agent; further investigations are needed to establish the hypoglycemic mechanism of the plant and the compound Cirsiliol $4^{\prime}$-O-glucoside.

\section{Data Availability}

The data used to support the findings of this study are available from the corresponding author upon request.

\section{Conflicts of Interest}

The authors declare that there are no conflicts of interest regarding the publication of this paper.

\section{Acknowledgments}

The authors acknowledge M. C. Ramiro Cruz-Durán for the plant determination and M. C. Christian Alan CabelloHernández for handling the animals at the Bioterium. This project was partially sponsored by DGAPA, PAPIIT IN228216, and PAPIIT IN226719.

\section{References}

[1] IDF Diabetes Atlas, Country Reports; Report Builder, Mexico, 8th Edition edition, 2017.

[2] American Diabetes Association, "Classification and diagnosis of diabetes," Diabetes Care, vol. 38, supplementary 1, pp. S8-S16, 2015.

[3] A. Espejo-Serna, A. R. López-Ferrari, N. Martínez-Correa, and V. A. Pulido-Esparza, "Bromeliad flora of Chiapas state, Mexico: richness and distribution," Phytotaxa, vol. 310, no. 1, article 1, 2017.

[4] F. Parada and C. Duque, "Studies on the aroma of Pinuela fruit pulp (Bromelia plumieri): free and bound volatile composition and characterization of some glucoconjugates as aroma precursors," Journal of High Resolution Chromatography, vol. 21, no. 10, pp. 577-581, 1998.

[5] A. Andrade-Cetto and A. E. Medina-Hernández, "Hypoglycemic effect of Bromelia plumieri (E. Morren) L.B. Sm., leaves in STZ-NA-induced diabetic rats," Frontiers in Pharmacology, vol. 4, 2013.

[6] N. Olaw, "Committee for the update of the guide for the care and use of laboratory animals," in Guide for the Care and Use of Laboratory Animals, Institute for Laboratory Animal Research, Division on Earth and Life Studies, and National Research Council, 8th edition, 2011.

[7] P. Masiello, C. Broca, R. Gross et al., "Experimental NIDDM: development of a new model in adult rats administered streptozotocin and nicotinamide," Diabetes, vol. 47, no. 2, pp. 224-229, 1998.

[8] A. Andrade-Cetto, S. M. Escandón-Rivera, G. M. TorresValle, and L. Quijano, "Phytochemical composition and chronic hypoglycemic effect of rhizophora mangle cortex on STZ-NAinduced diabetic rats," Revista Brasileira de Farmacognosia, vol. 27, no. 6, pp. 744-750, 2017.

[9] N. P. Rai, B. B. Adhikari, A. Paudel et al., "Phytochemical constituents of the flowers of Sarcococca coriacea of Nepalese origin," Journal of Nepal Chemical Society, vol. 21, pp. 1-7, 2006.

[10] R. Karthikeyan, C. Devadasu, and P. S. Babu, "Isolation, characterization, and RP-HPLC estimation of P-coumaric acid from methanolic extract of durva grass (Cynodon dactylon Linn.) (Pers.)," International Journal of Analytical Chemistry, vol. 2015, Article ID 201386, 7 pages, 2015.

[11] C. Lin, Y. Huang, L. Cheng, S. Sheu, and C. Chen, "Bioactive flavonoids from Ruellia tuberosa," National Research Institute of Chinese Medicine, vol. 17, no. 3, pp. 103-109, 2006.

[12] M. Hassan, H. Mohammed, A. Nageeb et al., "Phytochemical and pharmacological studies of Citharexylum quadrangulare Jacq. leaves," Journal of Medicinal Plants Research, vol. 10, no. 18, pp. 232-241, 2016.

[13] R. H. Delaporte, K. P. Guzen, A. Laverde Jr., A. R. dos Santos, and M. H. Sarragiotto, "Phenylpropanoid glycerols from Tillandsia streptocarpa Baker (Bromeliaceae)," Biochemical Systematics and Ecology, vol. 34, no. 7, pp. 599-602, 2006.

[14] O. Shirota, S. Sekita, M. Satake, Y. Ni, and H. Weiyi, "Chemical constituents of Chinese folk medicine 'Sân Léng', Sparganium stoloniferum," Journal of Natural Products, vol. 59, no. 3, pp. 242-245, 1996. 
[15] W. Li, K. Koike, Y. Asada, T. Yoshikawa, and T. Nikaido, "Biotransformation of low-molecular-weight alcohols by Coleus forskohlii hairy root cultures," Carbohydrate Research, vol. 338, no. 8, pp. 729-731, 2003.

[16] S. Li, M.-H. Pan, C.-Y. Lo et al., "Chemistry and health effects of polymethoxyflavones and hydroxylated polymethoxyflavones," Journal of Functional Foods, vol. 1, no. 1, pp. 2-12, 2009.

[17] L. M. Manetti, R. H. Delaporte, and A. Laverde Jr., "Metabólitos secundários da família bromeliaceae," Química Nova, vol. 32, no. 7, pp. 1885-1897, 2009.

[18] P. Sharma, M. Alam, and M. V. Chari, "Isolation and structure of isopropyl-a-o-glucoside from the coral Sclerophytum capitalis," Journal of Natural Products, vol. 52, no. 2, pp. 395-397, 1989.

[19] A. El Barky, S. A. Hussein, A.-E. Alm-Eldeen, A. Hafez, and T. Mohamed, "Review diabetes management saponins and their potential role in diabetes mellitus," 2017.

[20] N. Somsak, P. Peerawit, and T. Chusri, "Hypoglycemic activity in diabetic rats of stigmasterol and sitosterol-3-O- $\beta$-Dglucopyranoside isolated from Pseuderanthemum palatiferum (Nees) Radlk. leaf extract," Journal of Medicinal Plants Research, vol. 9, no. 20, pp. 629-635, 2015.

[21] V. Amalan, N. Vijayakumar, D. Indumathi, and A. Ramakrishnan, "Antidiabetic and antihyperlipidemic activity of pcoumaric acid in diabetic rats, role of pancreatic GLUT 2: In vivo approach," Biomedicine \& Pharmacotherapy, vol. 84, pp. 230-236, 2016.

[22] A. Abdel-Moneim, S. M. A. El-Twab, A. I. Yousef, E. S. A. Reheim, and M. B. Ashour, "Modulation of hyperglycemia and dyslipidemia in experimental type 2 diabetes by gallic acid and p-coumaric acid: The role of adipocytokines and PPAR $\gamma$," Biomedicine \& Pharmacotherapy, vol. 105, pp. 1091-1097, 2018. 


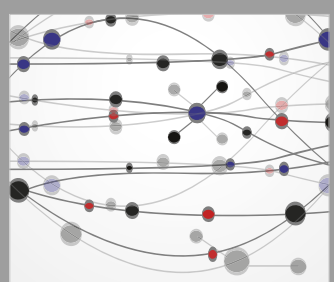

The Scientific World Journal
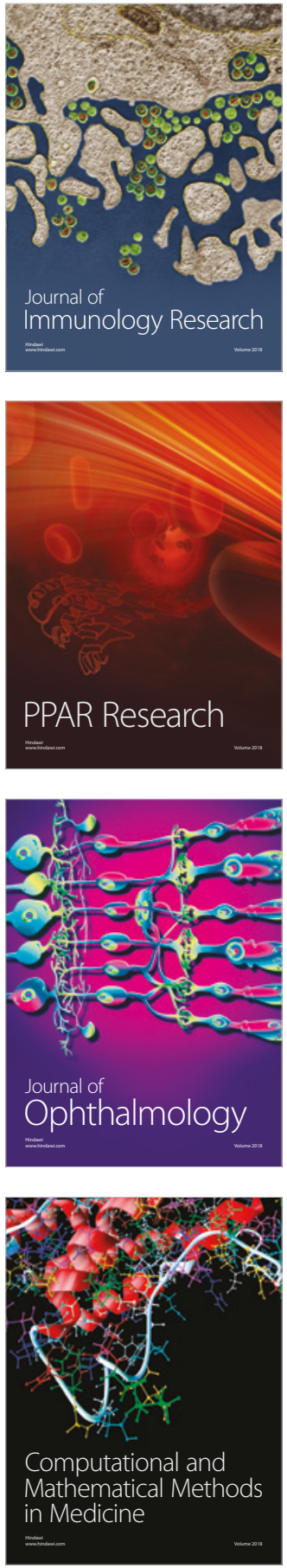

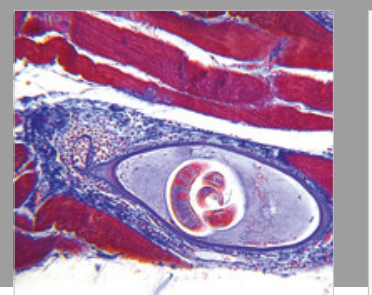

Gastroenterology Research and Practice

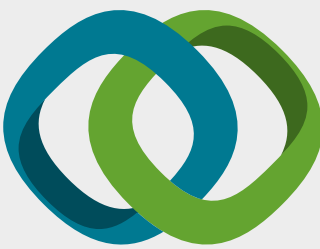

\section{Hindawi}

Submit your manuscripts at

www.hindawi.com
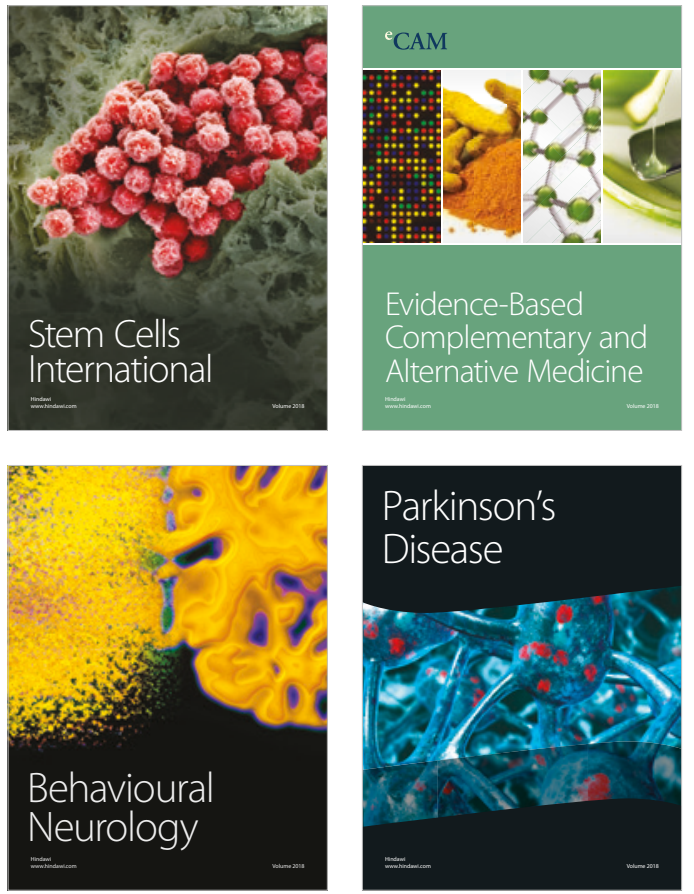

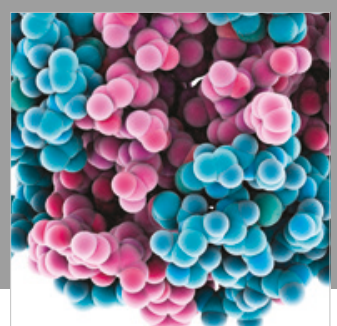

ournal of

Diabetes Research

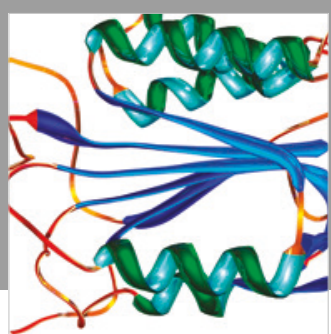

Disease Markers
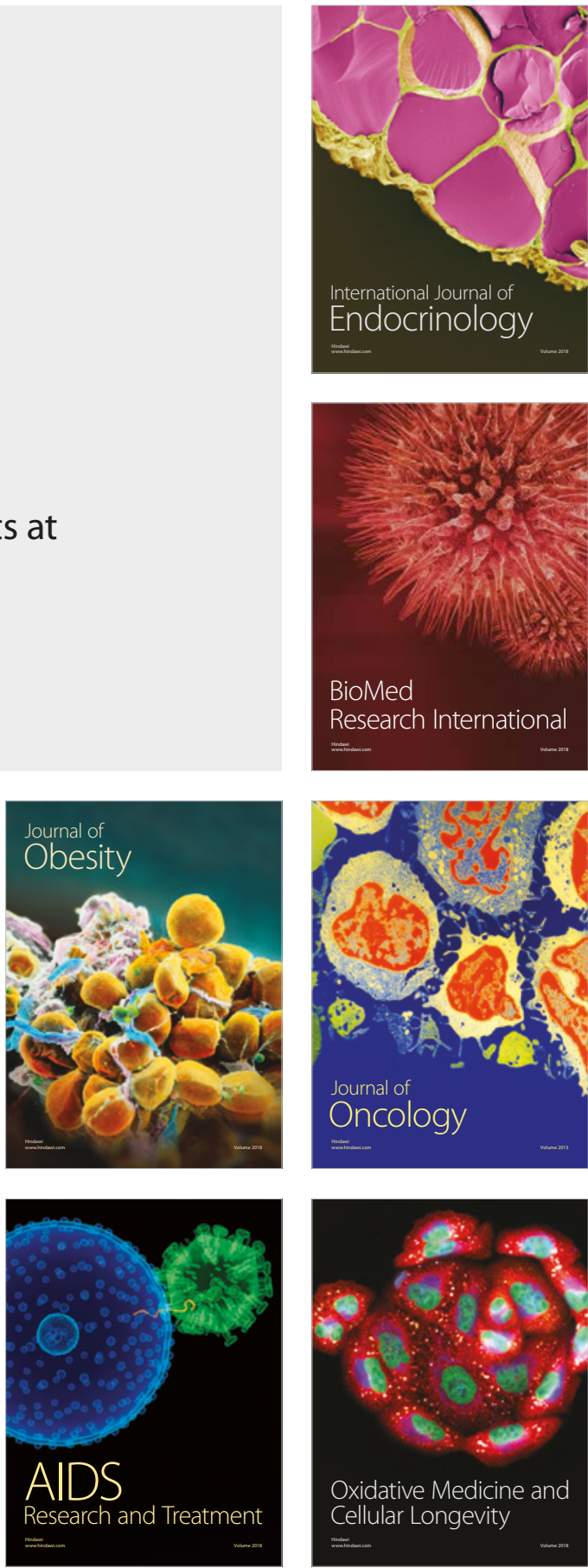